\title{
Comparison of the usefulness of a targeted temperature management protocol before and after modification: A single- centre retrospective study
}

\section{Trauma and Emergency Medical Centre, Medical Hospital of Tokyo Medical and Dental University Kanae Ochiai, Yasuhiro Otomo}

\section{【Background】}

ERC guidelines 20151):

- TTM is recommended for adults after OHCA with an initial shockable rhythm and is suggested for those with an initial nonshockable rhythm who remain unresponsive after ROSC.

- Maintain a constant, target temperature between $32^{\circ} \mathrm{C}$ and $36^{\circ} \mathrm{C}$ for at least 24 hours.

Nielsen et al. reported that hypothermia at a targeted temperature of $33^{\circ} \mathrm{C}$ did not confer a benefit as compered with a targeted temperature of $36^{\circ} \mathrm{C}^{2)}$.

\section{$35^{\circ} \mathrm{C}$ protocol used in our institute}

All the patients who do not fulfill exclusion criteria are included Exclusion criteria

Glasgow Coma Scale Motor: 6

Patients who do not receive treatment in our department

Patients with total brain ischemia in CT scan

Withdrawal of treatment

Method

Maintain at $35^{\circ} \mathrm{C}$ for 24 hours using surface cooling pad device or heat exchanger attached to ECMO circuit

Rewarm by $0.25^{\circ} \mathrm{C} /$ hour or slower

\section{【Method】}

OHCA patients aged $\geq 18$ years who were transported to a tertiary hospital in Tokyo were included.

We compared the $35^{\circ} \mathrm{C}$ protocol cohort (December 2017 to May 2019) with the conventional protocol cohort (June 2015 to

November 2017) in terms of performance rate, background factors, and outcomes.

【Result】

OHCA patients: $\mathrm{n}=337$

Performance rate of conventional protocol: 35/209 (16.7\%)

Performance rate of $35^{\circ} \mathrm{C}$ protocol: $42 / 128$ (32.8\%)

Temperature transition

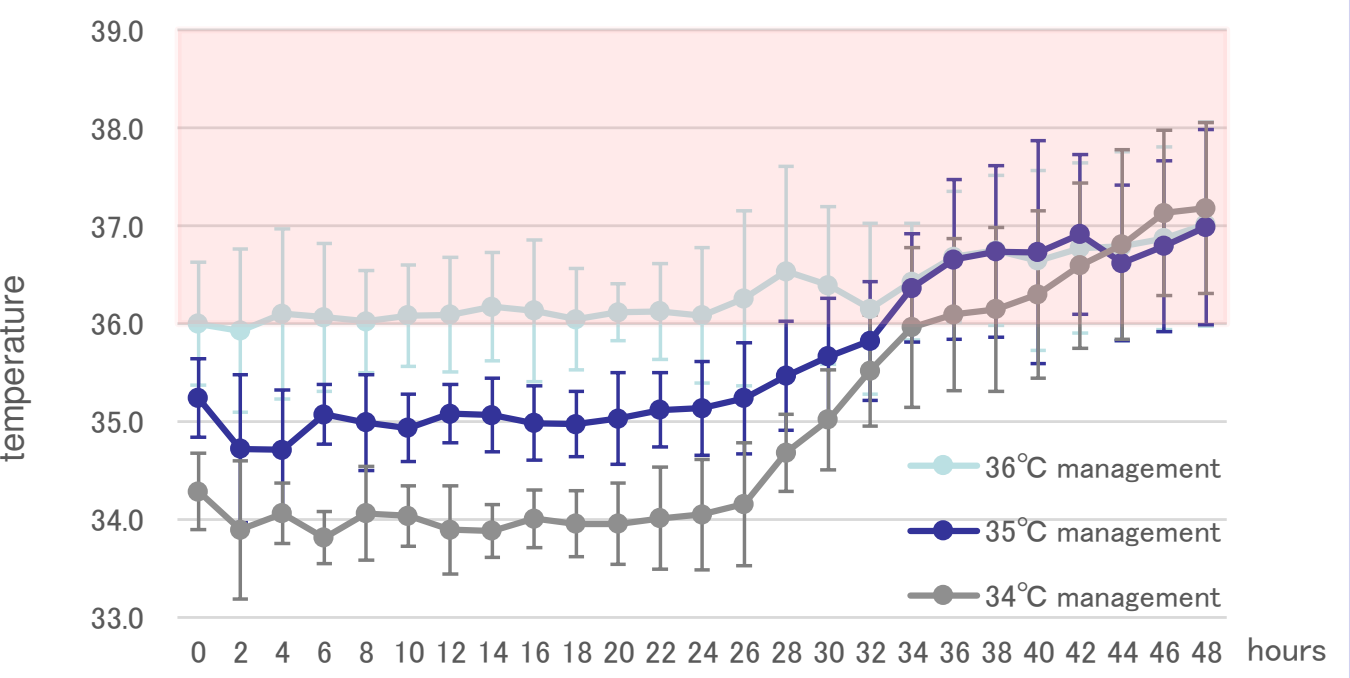

\begin{tabular}{|c|c|c|c|}
\hline & & $\begin{array}{l}\text { Conventional } \\
\qquad n=34\end{array}$ & $\begin{array}{l}35^{\circ} \mathrm{C} \\
\mathrm{n}=32\end{array}$ \\
\hline Age & Median (IQR) & $59(53,70)$ & $64(53,73)$ \\
\hline Sex Male & $\mathrm{n}(\%)$ & $29(85.3)$ & $24(75.0)$ \\
\hline Witness & Yes n (\%) & $29(85.3)$ & $28(87.5)$ \\
\hline Bystander CPR & Yes n (\%) & $25(73.5)$ & $20(62.5)$ \\
\hline Initial rhythm Shockable & $\mathrm{n}(\%)$ & $28(82.4)$ & $15(46.9)$ \\
\hline Transfer time (minute) & Median (IQR) & $6(5,8)$ & $6(4,7)$ \\
\hline GCS Motor after ROSC & Median (IQR) & $1(1,3)$ & $1(1,1)$ \\
\hline $\begin{aligned} & \text { Rhythm on arrival } \text { Shockable } \\
& \text { PEA } \\
& \text { Asystole } \\
& \text { ROSC }\end{aligned}$ & n (\%) & $\begin{array}{c}8(23.5) \\
4(11.8) \\
3(8.8) \\
19(55.9)\end{array}$ & $\begin{array}{c}1(3.1) \\
8(25.0) \\
6(18.8) \\
17(53.1)\end{array}$ \\
\hline pH after ROSC (ABG) & Median (IQR) & $7.194(7.069,7.278)$ & $6.992(6.898,7.276)$ \\
\hline Lactate after ROSC (ABG) & Median (IQR) & $9.0(7.3,13.0)$ & $10.5(7.7,17.0)$ \\
\hline Cardiogenic arrest & Yes n (\%) & $28(82.4)$ & $23(71.9)$ \\
\hline ECPR & Yes n (\%) & $4(11.8)$ & $8(25.0)$ \\
\hline Intra-aortic balloon pumping & Yes n (\%) & $6(17.6)$ & $7(21.9)$ \\
\hline Coronary angiography & Yes n (\%) & $23(67.6)$ & $21(65.6)$ \\
\hline TTM completed & Yes n (\%) & $28(82.4)$ & $25(78.1)$ \\
\hline $\begin{array}{l}\text { Cooling method Surface } \\
\qquad \text { Intravenous }\end{array}$ & $\mathrm{n}(\%)$ & $\begin{array}{c}30(88.2) \\
4(11.8)\end{array}$ & $\begin{array}{l}25(78.1) \\
7(21.9)\end{array}$ \\
\hline ROSC to TT time (minute) & Median (IQR) & $426(300,493)$ & $335(233,441)$ \\
\hline $\begin{array}{l}\text { Circulatory complications } \\
\text { Bleeding } \\
\text { Electrolytes disturbance }\end{array}$ & $\mathrm{n}(\%)$ & $\begin{array}{c}10(29.4) \\
3(8.8) \\
6(17.6)\end{array}$ & $\begin{array}{l}8(25.0) \\
2(6.3) \\
9(28.1)\end{array}$ \\
\hline Mortality Survive & $\mathrm{n}(\%)$ & $25(73.5)$ & $23(71.9)$ \\
\hline $\begin{array}{l}\text { Neurological outcome } \\
\text { Favorable }\end{array}$ & $\mathrm{n}(\%)$ & $15(44.1)$ & $11(34.4)$ \\
\hline
\end{tabular}

【Conclusion】

The new $35^{\circ} \mathrm{C}$ protocol can be more readily applied than the conventional protocol, without any increase in complications or mortality.

1) Resuscitation. 2015, 95: 202-22

2) N Engl J Mad. 2013 5; 369(23): 2197-206
TTM targeted temperature management OHCA out of hospital cardiac arrest ROSC return of spontaneous circulation ECMO extra-corporeal membrane oxygenation

CPR cardiopulmonary resuscitation

PEA pulseless electrical activity

ECPR extracorporeal cardiopulmonary resuscitation

TT target temperature

\section{$\begin{array}{ll}\text { I have no financial relationships to disclose } & \begin{array}{l}\text { E } \\ \mathbf{R}\end{array} \\ \end{array}$}

